\title{
Power System Mode optimization by piecewise-linear approximation of energy characteristics of Power Plants
}

\author{
Tulkin Gayibov ${ }^{1, *}$, Sherxon Latipov ${ }^{1}$, and Bakhadir Uzakov ${ }^{2}$ \\ ${ }^{1}$ Tashkent State Technical University named after Islam Karimov, Uzbekistan \\ ${ }^{2}$ Karakalpak State University, Uzbekistan
}

\begin{abstract}
The issues of improving of methods and algorithms for power system short-term mode optimal planning are considered. The main disadvantages and calculation difficulties typical for traditional algorithms are described. An effective algorithm for power system mode optimization based on a piecewiselinear approximation of nonlinear dependencies is proposed. Application of the proposed algorithm can effectively increase the accuracy and overcome many calculation difficulties associated with discontinuity of energy characteristics of power plants and various constraints in the form of equality and inequality.
\end{abstract}

\section{Introduction}

Solving the problem of short-term mode optimization of the power system provides definition for each interval of the considered planning period the active loads of the power plants, which ensure reliable supply of high-quality electricity to all consumers with minimal costs for their generation and transmission. Plan development is usually carried out on the basis of solving the corresponding optimization problem, where costs are taken into account by the objective function, and the requirements for the quality of electricity and reliability of power supply are taken into account by simple and functional constraints in the form of equality and inequality. Such problem for the modern power systems presents the difficult nonlinear mathematical programming problems, characterized by the presence of discontinuities in the energy characteristics of the power plants involved in optimization, as well as the presence of a large number of various constraints in the form of equality and inequality.

The most widespread and widely practically used algorithms for such problem solution in basis of gradient methods [1-5] and Newton's method [6-8] provide for the approximation of the energy characteristics of power plants by polynomials of various degrees. Application of a quadratic polynomial facilitates convenient solution of the problem. However, due to the low approximation accuracy a result can be obtained with a significant error, reducing optimization effect. With increase of the approximating polynomial degree, the approximation accuracy and, correspondingly, optimization increase as well. But the problem can become multi-extreme, which solution is associated with additional difficulties.

Another problem in the problem solution is related to taking into account the constraints in the form of inequality.
Currently, in the power system mode optimization algorithms based on the gradient methods [1-5], simple constraints imposed on adjustable parameters are taken into account quite effectively, fixing in each iteration corresponding adjustable parameters within the violated limits. Taking into account of complex functional constraints in them and in the optimization algorithms by the Newton's method [6-8] is carried out by the penalty function method, characterized by a number of advantages such as universality, ease of implementation, and the calculation possibility from any initial approximation. At the same time, in some cases, when the penalty factors increase in these algorithms, computational difficulties arise due to the deterioration and thereby loss of reliability of optimization process convergence. The same difficulty occurs in the problem solution by the method of incremental rates equality $[8,9]$. In [10], by the example of the electric network mode optimization, it was shown that in cases of application of the straight-linear approximation of the objective function, the iterative process converges is slow and unreliable.

In this regard, the problem of effective algorithm development and implementation for power system mode optimization with effective consideration of the real energy characteristics of power plants and constrains remains actual.

\section{The optimization algorithm}

In the problems with a large number of simple and complex constraints and with discontinuous energy characteristics of plants, the power system mode optimization can be effectively solved by the method of successive piecewiselinear approximation of nonlinear dependencies. In this case,

* Corresponding author: tulgayibov@gmail.com 
the nonlinear programming problem is reduced to the linear programming one and is solved by the simplex method.

In this paper, we present an power system mode optimization algorithm based on a piecewise-linear approximation of the nonlinear energy characteristics of power plants and constraint functions free from the indicated calculation difficulties.

To reveal the essence of the algorithm, we consider the problem of the power system active load optimal distribution between thermal power plants (TPPs), taking into account the constraints, which is formed as follows:

minimize the function of the total fuel equivalent consumption in TPPs

$$
B=\sum_{i=0}^{n} B_{i}\left(P_{i}\right)
$$

subject to constrains

$$
\begin{aligned}
& W(P)=\sum_{i=1}^{n} P_{i}-P_{H}-\pi=0, \\
& h_{l}(P) \leq h_{l s .}, \quad l=1,2, . L \\
& P_{i}^{\min } \leq P_{i} \leq P_{i}^{\max }, \quad i=1,2, . . n,
\end{aligned}
$$

where $n$ is the number of rated power plants; $P_{i}$ is the active load of the $i$-th TPP; $B_{i}\left(P_{i}\right)$ is equivalent fuel consumption in $i$-th TPP at its load $P_{i} ; P_{L}$ is the total load of the power system; $\pi$ is the total active power loss in electric networks of the power system; $W(P)$ is the unbalance active power function in power system; $h_{l}, h_{l s}$ are the calculated and specified limit values of the $l$-th function of the load of power plants (for example, the active power flow through a controlled transmission line).

The main condition under which the proposed algorithm can be applied is the separability of functions, i.e. the possibility of presenting them as a sum of several functions, each of which depends on only one variable [11]. The objective function in the adopted optimization model for the power system mode is always separable. Functional constraints for which these conditions are not satisfied are preliminarily reduced to a separable form using special methods, as in [11].

Normally, in the iterative calculation optimization cycle, the value of the total active power loss in electric networks is taken from the calculation of its steady state mode, and therefore the constraint (2) is linear. (3) represents a lot of constraints in the form of inequality on active power flow in some monitored transmission lines, determined from the conditions of ensuring the static stability of the power system, and on the permissible currents of individual transmission lines. The flows of active power in monitored lines in the proposed algorithm is expressed through the distribution coefficients, which determine their values with sufficient accuracy for practical purposes. As a result, these constraints turn into a linear form.

During optimization by this algorithm, a piecewiselinear approximation of the objective function and nonlinear functional constraints is made by choosing in a given interval $\left[P_{i, \min } ; P_{i, \max }\right] m_{i}$ points with coordinates $\left[B_{i k} ; P_{i k}\right]$ and replacing it in each segment $\left[P_{i, k} ; P_{i, k+1}\right]$ by the linear functions:

$$
\bar{B}_{i k}\left(P_{i}\right)=B_{i}\left(P_{i k}\right)+\frac{B_{i}\left(P_{i k+1}\right)-B_{i}\left(P_{i k}\right)}{P_{i k+1}-P_{i k}}\left(P_{i}-P_{i k}\right)
$$

$$
\bar{h}_{i k}(P)=h_{i}\left(P_{i k}\right)+\frac{h_{i}\left(P_{i k+1}\right)-h_{i}\left(P_{i k}\right)}{P_{i k+1}-P_{i k}}\left(P_{i}-P_{i k}\right) .
$$

representing the values $P_{i}$ in the interval $\left[P_{i k} ; P_{i k+1}\right]$ as

$$
P_{i}=\lambda_{k} P_{i k}+\lambda_{k+1} P_{i k+1}
$$

and choosing $\lambda_{k}$ and $\lambda_{k+1}$ under the condition

$\lambda_{k}+\lambda_{k+1}=1$, we obtain the following approximate linear programming problem for (1)-(4):

minimize function

$$
B=\sum_{i=1}^{n} \sum_{k=1}^{m i} \lambda_{i k} \bar{B}_{i k}\left(P_{i}\right)
$$

subject to constraints

$$
\left.\begin{array}{l}
\bar{W}=\sum_{i=1}^{n} \sum_{k=1}^{m i} \lambda_{i k} W_{i k}(P)=0, \\
\bar{h}_{i}=\sum_{i=1}^{n} \sum_{k=1}^{m i} \lambda_{i k} \bar{h}_{i k}(P) \leq 0, \quad i=1,2, . L, \\
\sum_{k=1}^{m i} \lambda_{i k}=1 ; \quad i=1,2, . n, \\
\lambda_{i k} \geq 0 ; \quad i=1,2, . n \quad k=1,2, . m_{i} .
\end{array}\right\}
$$

where $\lambda_{i k}$ are the unknown parameters of the obtained linear programing problem.

Problem (6)-(7) is solved by the simplex method, taking into account constraints on the basis choice, which consists of inadmissibility of including two vectors in the basis that correspond to two non-neighboring ones.

After solving the problem (6)-(7), approximate optimal values of TPP loads $P_{i}$ are determined by (5). If it is necessary, in order to find the exact solution, we can perform the next step with a more accurate piecewise-linear approximation of the nonlinear dependences of the separable problem in the vicinity of the point obtained in the previous step.

\section{The results of calculation experiments}

We illustrate the computational qualities of the algorithm described above by optimizing of mode of the power system with testing circuit [8] shown in Fig. 1, where 0 (balancing), 1, 6, 7 are nodes with TPPs.

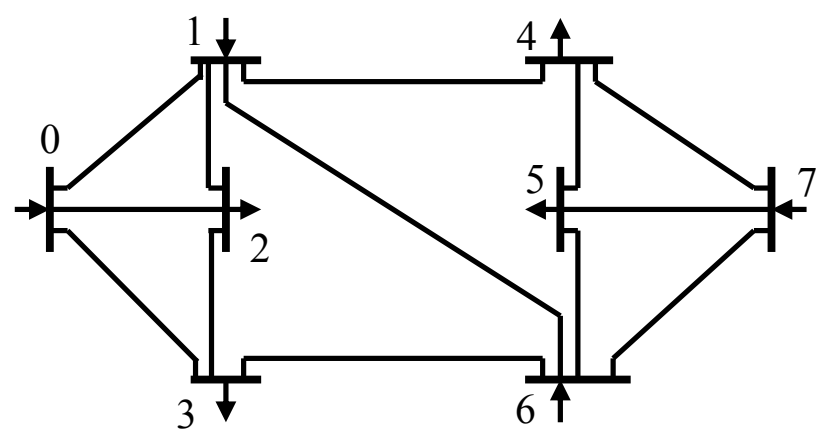

Fig. 1. The scheme of the power system

The characteristics of the incremental rates of the fuel equivalent consumption of these TPPs are given in table 1 . Where $B_{00}, B_{10}, B_{60}, B_{70}$ are fuel equivalent consumptions 
per hour at minimum loads of TPPs. The power of the load nodes are, MW: $P_{2}=400, P_{3}=600, P_{4}=200, P_{5}=500$. In three power transmission lines (PTLs) the power flows are limited, MW: $P_{6-3} \leq 450, \quad P_{6-5} \leq 150, \quad P_{0-3} \leq 100$. Matrices of power distribution coefficients of the nodes through these lines are given in table 2 .

Table 1. Characteristics of the incremental rate of fuel equivalent consumption in TPPs.

\begin{tabular}{|c|c|c|c|c|c|c|c|}
\hline \multicolumn{2}{|c|}{$\begin{array}{c}\text { ТPР }-0 \\
B_{00}=114 t . f . e . / h .\end{array}$} & \multicolumn{2}{|c|}{$\begin{array}{c}\text { TPP }-1 \\
B_{10}=32 \text { t.f.e. } / \text { h. }\end{array}$} & \multicolumn{2}{|c|}{$\begin{array}{c}\text { ТPР }-6 \\
B_{60}=114,8 \text { t.f.e. } / \text { h. }\end{array}$} & \multicolumn{2}{|c|}{$\begin{array}{c}\text { ТРР }-7 \\
B_{70}=97 \text { t.f.e. } / \text { h. }\end{array}$} \\
\hline $\begin{array}{c}b_{0} \\
\text { t.f.e. } / M W . h \\
\end{array}$ & $\begin{array}{c}P_{0} \\
M W \\
\end{array}$ & $\begin{array}{c}b_{1} \\
\text { t.f.e./MW.h } \\
\end{array}$ & $\begin{array}{c}P_{1} \\
M W\end{array}$ & $\begin{array}{c}b_{6} \\
\text { t.f.e./MW.h }\end{array}$ & $\begin{array}{c}P_{6} \\
M W\end{array}$ & $\begin{array}{c}b_{7} \\
\text { t.f.e. } / M W . h\end{array}$ & $\begin{array}{c}P_{7} \\
M W\end{array}$ \\
\hline 0,316 & 320 & 0,34 & 70 & 0,2705 & 400 & 0,22 & 320 \\
\hline 0,317 & 384 & 0,359 & 77 & 0,2836 & 565 & 0,27 & 398 \\
\hline 0,319 & 427 & 0,366 & 92 & 0,2967 & 583 & 0,295 & 438 \\
\hline 0,334 & 428 & 0,524 & 92 & 0,3098 & 595 & 0,32 & 450 \\
\hline 0,3341 & 640 & 0,533 & 100 & 0,3229 & 647 & 0,345 & 495 \\
\hline & & & & 0,336 & 715 & 0,395 & 502 \\
\hline & & & & 0,3491 & 759 & & \\
\hline & & & & 0,3622 & 790 & & \\
\hline
\end{tabular}

Table 2. Power distribution coefficients of the nodes through controlled PTLs.

\begin{tabular}{|c|c|c|c|c|c|c|c|}
\hline \multirow{2}{*}{ PTL } & \multicolumn{7}{|c|}{ Nodes } \\
\cline { 2 - 8 } & 1 & 2 & 3 & 4 & 5 & 6 & 7 \\
\hline $6-3$ & 0,2536 & $-0,0713$ & $-0,172$ & 0,4106 & 0,439 & 0,6343 & 0,4986 \\
\hline $6-5$ & $-0,0701$ & 0,02025 & 0,0484 & $-0,281$ & $-0,4223$ & 0,1029 & $-0,2986$ \\
\hline $0-3$ & $-0,14$ & $-0,1735$ & $-0,267$ & $-0,1744$ & $-0,1917$ & $-0,224$ & $-0,1939$ \\
\hline
\end{tabular}

For comparison, presented the reference result of the optimal distribution of the power system total load between TPPs taking into account the given simple and functional constraints:

$P_{0}=545,023 \mathrm{MW} ; P_{1}=70 \mathrm{MW} ; P_{6}=634,977 \mathrm{MW} ; P_{7}=$ $450 \mathrm{MW} ; P_{6-3}=450 \mathrm{MW} ; P_{6-5}=150 \mathrm{MW} ; P_{0-3}=68,5$

MW; $B=532,57$ t.e.f. $/$ h.,

where t.f.e./h. is tones of fuel equivalent per hour.

By numerical integration of incremental rate characteristics of TPPs at given points (table 1), we perform a piecewise-linear approximation of fuel equivalent consumption characteristics of TPPs. As a result, the function of total fuel equivalent consumption in power system is expressed as follows:

$$
\begin{aligned}
& \bar{B}=114 \lambda_{11}+134,56 \lambda_{12}+147,93 \lambda_{13}+148,256 \lambda_{14}+219,75 \lambda_{15}+ \\
& +32 \lambda_{21}+34,446 \lambda_{22}+39,883 \lambda_{23}+39,883 \lambda_{24}+44,112 \lambda_{25}+ \\
& +97 \lambda_{31}+116,11 \lambda_{32}+127,41 \lambda_{33}+131,1 \lambda_{34}+146,062 \lambda_{35}+ \\
& +148,652 \lambda_{36}+44,8 \lambda_{41}+90,513 \lambda_{42}+95,736 \lambda_{43}+111,505 \lambda_{44}+ \\
& +133,017 \lambda_{45}+148,069 \lambda_{46}+158,403 \lambda_{47}
\end{aligned}
$$

We express the loads of individual TPPs as

$$
\left.\begin{array}{rl}
P_{0} & =320 \lambda_{01}+384 \lambda_{02}+427 \lambda_{03}+428 \lambda_{04}+640 \lambda_{05}, \\
P_{1} & =70 \lambda_{11}+77 \lambda_{12}+92 \lambda_{13}+92 \lambda_{14}+100 \lambda_{15}, \\
P_{6} & =320 \lambda_{61}+398 \lambda_{62}+438 \lambda_{63}+450 \lambda_{64}+495 \lambda_{65}+502 \lambda_{66} \\
P_{7} & =400 \lambda_{71}+565 \lambda_{72}+583 \lambda_{73}+595 \lambda_{74}+647 \lambda_{75}+ \\
& +715 \lambda_{74}+759 \lambda_{75}
\end{array}\right\}
$$

$$
\begin{aligned}
& \lambda_{01}+\lambda_{02}+\lambda_{03}+\lambda_{04}+\lambda_{05}=1, \\
& \lambda_{11}+\lambda_{12}+\lambda_{13}+\lambda_{14}+\lambda_{15}=1, \\
& \lambda_{61}+\lambda_{62}+\lambda_{63}+\lambda_{64}+\lambda_{65}+\lambda_{66}=1, \\
& \lambda_{71}+\lambda_{72}+\lambda_{73}+\lambda_{74}+\lambda_{75}+\lambda_{76}+\lambda_{77}=1 .
\end{aligned}
$$

Substituting these expressions of the power plant loads into the power balance equation in power system and the formulas for the power flows along the controlled PTLs expressed through the given distribution coefficients, we obtain a linear programming problem that is effectively solved by the simplex method. Then, substituting the obtained values of the variables $\lambda_{i k}$ into expressions (9), we find the optimal loads of TPPs:

$P_{0}=541,487 \mathrm{MW} ; \quad P_{1}=70 \mathrm{MW} ; \quad P_{6}=621,985 \mathrm{MW}$; $P_{7}=466,528 \mathrm{MW}$.

At these loads of TPPs we have:

$P_{6-3}=450 \mathrm{MW} ; \quad P_{6-5}=150 \mathrm{MW} ; \quad P_{0-3}=68,4 \mathrm{MW} ;$ $B=532,68$ t.f.e./h.

Having compared the obtained solution with the reference result, it can be argued that there is no need to perform the following optimization step for its refinement. At the same time, the TPPs loads turned out to be quite close to the reference ones, the fuel equivalent consumption overrun is negligible.

Improving the piecewise-linear approximation accuracy of the problem can be achieved by increasing the number of given points in incremental rate characteristics of fuel equivalent consumption. In this case, the dimension of the resulting linear programming problem increases accordingly.

subject to conditions 
On the basis of numerous experimental calculations, it has been established that in real-life problems of power system mode optimization, it is expedient to carry out a calculation with piecewise-linear approximation of nonlinear dependencies using all the given points in incremental rate characteristics of fuel equivalent consumption of power plants. This enables to ensure the absolute convergence of calculation process and obtain the optimal result in one step with sufficient accuracy.

Another positive feature of the described algorithm is that it successfully overcomes the difficulties associated with the discontinuity of the energy characteristics of power plants.

\section{Conclusion}

Optimization of power system modes by piecewise-linear approximation of nonlinear dependencies allows us to consider effectively the simple and functional constraints in the form of equality and inequality.

In optimization of a power system mode by piecewiselinear approximation of nonlinear dependences, difficulties associated with the discontinuities in energy characteristics of power plants are automatically overcome.

\section{References}

1. Methods for power system mode optimizaion. V.M. Gornstein, B.P. Miroshnichenko, A.V. Ponomarev and others; Edited by V.M.Gornstein (Energy, Moscow, 1981).
2. Krumm L.A. Methods of optimization and control of electric power systems (Nauka, Novosibirsk, 1980).

3. Automation of dispatch control in the electric power systems / Edited by Yu.N. Rudenko and V.A. Semenov (Publishing MPEI, Moscow, 2000).

4. Murty, P. S. R., Optimization and Control in Power Systems (BS Publications, Headarabad, 2008).

5. M.R. Irving and Y.H. Song. Optimization Methods for Electric Power Systems, Part 1, Mathematical Optimization Methods. IEE Power Engineering Journal, 14 (5): 245-254, (2000).

6. D.I. Sun, B. Ashley, B. Brewer, A. Hughes and W.F. Tinney. Optimal Power Flow by Newton Approach. IEEE Trans. Power Apparatus and Systems, PAS-103(2): 2864 2878, (1984).

7. Arzamastsev D.A., Bartolomei P.I., Kholyan A.M. ACS and optimization of power system mode: Textbook for university students (Higher school, Moscow, 1983).

8. Fazylov H.F., Nasyrov T.Kh. Steady state mode of power systems and its optimization (Moliya, Tashkent, 1999).

9. Nasirov T.Kh., Gayibov T.Sh. Theoretical foundations of power system mode optimization (Fan va texnologiya, Tashkent, 2014).

10. Gayibov T.Sh., Gafurov T.F. Problems of Energy and Resource Saving, No 2-3, 16-23 (2005).

11. G.V. Reklaitis, A. Ravindran, K. Ragsdel. Engineering optimization. Methods and Applications (John Wiley and Sons, NY, 1983). 